\title{
Stellar Populations of Brightest Cluster Galaxies and Intracluster Light
}

\author{
Louise O. V. Edwards and Isabella L. Trierweiller \\ Department of Astronomy, Yale University, \\ New Haven, CT USA \\ email: louise.edwards@yale.edu
}

\begin{abstract}
We present 3 representative cases from a sample of 16 local Brightest Cluster Galaxies observed using integral field spectroscopy. The observations extend to nearby neighbours and into the Intracluster Light (ICL). Population synthesis modeling shows that the ICL is younger and more metal poor compared to the BCG core and outskirts. This is consistent with a scenario in which the ICL grows by cluster processes, and alongside the growth of the BCG.
\end{abstract}

Keywords. galaxies: clusters: individual (Abell 85, Abell 2457, IIZw108), galaxies: cD, galaxies: evolution, galaxies: interactions, galaxies: stellar content

\section{Introduction and Sample Description}

Brightest Cluster Galaxies (BCGs) are dominated by old, red stellar populations. However, BCGs are still evolving. In cool core clusters, striking evidence for current star formation exists (e.g., Crawford et al. 1999; Edwards et al. 2007; McDonald et al. 2012) and many continue to accumulate mass through recent and ongoing mergers (e.g., Brough et al. 2011; Inagaki et al. 2015; Oliva-Altamirano et al. 2015). Spectroscopic observations of the Intracluster Light, which overlaps with the BCG outskirts, has been obtained for only a few systems (Melnick et al. 2012; Coccatto et al. 2010; Coccatto et al. 2011). Our goal is to determine the stellar populations of the BCG's core, outskirts, companions and the ICL concurrently. We use the SparsePak IFU (Bershady et al. 2004) on the WIYN telescope to collect spectra over a sample of nearby X-ray bright cluster BCGs. The setup is illustrated in Figure 1.

\section{First Results}

\subsection{Populations}

In all three of our galaxies the ICL shows much younger ages and lower metallicities (Z) than any region of the BCG (Figure 2). The core of the BCG typically has the oldest, most metal rich population. All fiber spectra within key regions are median combined achieving spectra with $\mathrm{S} / \mathrm{N} \sim 30$. Dwarf galaxies may pollute the ICL region, but as over 70 individual spectra are median combined together, we expect our final spectrum to be dominated by the envelope of ICL stars. Our high $\mathrm{S} / \mathrm{N}$ region spectra are then fit to model spectra from Walcher et al. (2009), which allow for $\alpha$-enhanced populations, using the STARLIGHT population synthesis code of Cid Fernandes et al. (2005). We use 54 base models which include ages ranging from $3 \mathrm{Gyr}-13 \mathrm{Gyr}$ and $\mathrm{Z}$ of $0.006,0.02,0.032$. We use the Chabrier (2003) initial mass function and the Calzetti et al. (2000) reddening law. Errors are estimated by finding the standard deviation $(\sigma)$ of solutions obtained from 5 sets of fits - each differing by randomly removing $5-10 \%$ of the fibers from the median average and re-running the model fitting. We find that a 1-2\% uncertainty in age, 

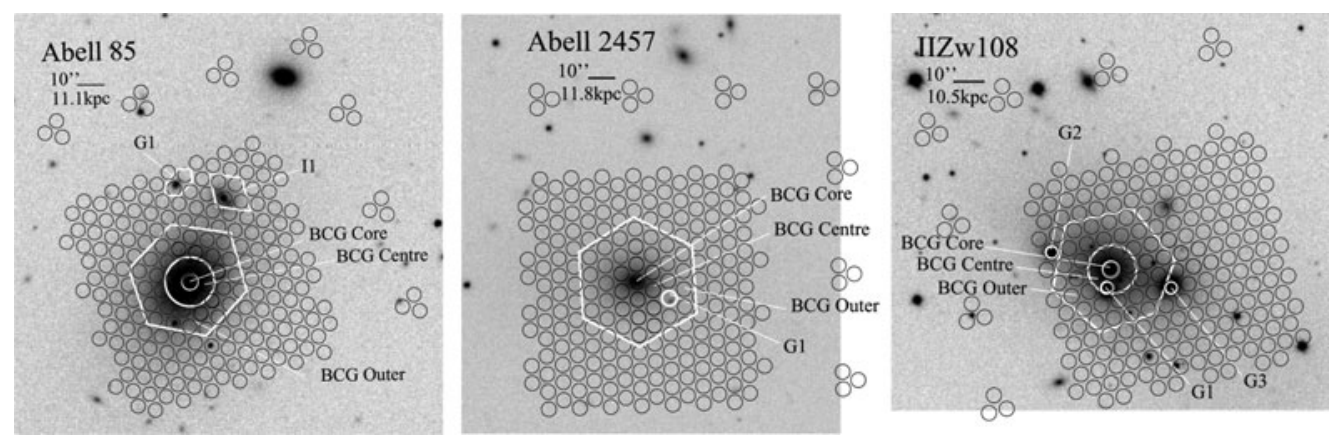

Figure 1. The field of view covered by SparsePak is illustrated on top of greyscale SDSS r' images. White polygons illustrate the fibres combined together to construct the region spectra. Interloping galaxies are delineated with an I, and spectroscopically confirmed cluster companion galaxies are labeled with a G. North is up with East to the left.
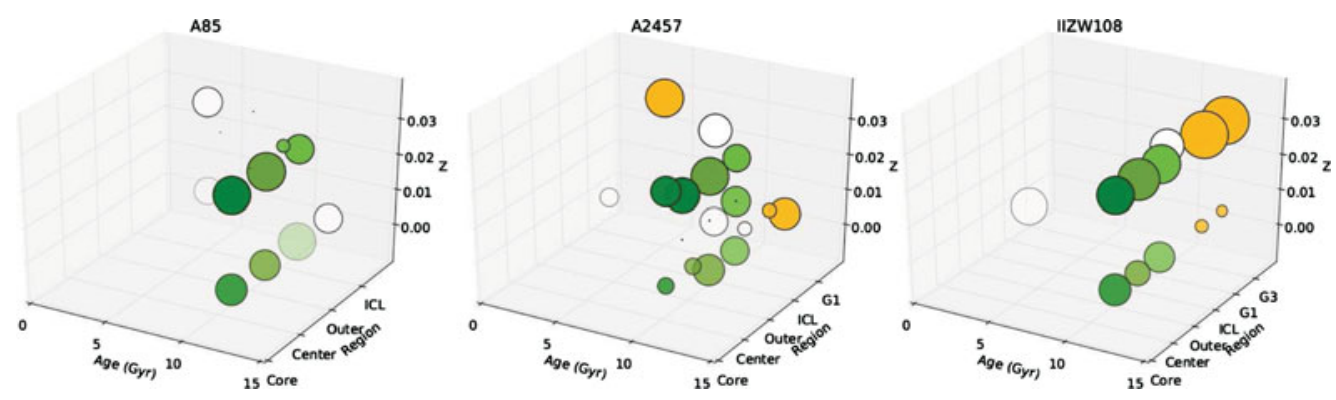

Figure 2. The results use the $\alpha$-enahnced models of Walcher et al. (2009). The $\chi^{2}$ of the fit to our spectrum is $\sim 0.4$ for the BCG core (dark grey), $\sim 0.6$ for the center and outer regions (medium grey) and $\sim 1.0$ for the ICL (white). The age and $\mathrm{Z}$ for the top 4 populations that make up the best-fit models are presented and the circle size represents the fraction of that population. Companion galaxies are shown if they are found to have a high likelihood of merging (light grey).

and $2-7 \%$ uncertainty in $\mathrm{Z}$ are derived from variation in the models used. The values of age and $\mathrm{Z}$ vary quite a bit when using different models, but we found the overall trends to be consistant when the spectra were fitted with Coelho et al. (2007) and Bruzual \& Charlot (2003) models. We can learn more by examining the relative fraction of the most abundant populations in each cluster, shown in Figure 2.

\subsection{Kinematics}

The velocity and velocity dispersion from the best-fit model are used to estimate the merging activity surrounding the BCG. The velocity dispersion corrected for instrumental broadenning, and the effective radius, measured off of the SDSS images, are used to calcuate the dynamical mass following Cappellari et al. (2006). Subsequently, we calculate the probability that the spectroscopic companions are bound to the BCG, as in Beers et al. (1982) and Brough et al. (2006). The companion to A85 has only a $25 \%$ likelihood of being bound, however, for A2457, the small projected distance and proximity in velocities $\left(11 \pm 10 \mathrm{~km} \mathrm{~s}^{-1}\right)$ produce a $93 \%$ likelihood. In IIZW108, G1 and G3 have a 95\% and $54 \%$ likelihood of being bound, with G2 having only a $20 \%$ probability as the velocity difference between it and the BCG is large $\left(390 \pm 10 \mathrm{~km} \mathrm{~s}^{-1}\right)$. For neighbours that have greater than a $50 \%$ likelihood of being bound, we use the relative velocity of the BCG and the companion to estimate the merger time by calculating the dynamical friction timescale (Binney \& Tremaine 1987). 


\section{Conclusions and Project Status}

In A85, there are no bound close companions, the optical light is smooth and the X-ray is relaxed as this is a cool core cluster. The BCG has no young metal poor component, while the ICL is dominated by young stars, with high and low metallicities. This is consistent with a scenario where infalling galaxies far from the cluster core have collected onto the ICL. A2457 has one bound companion, where the merging timescale is $2 \mathrm{Myr}$. The BCG is smooth in the optical but there exists X-ray substructure (Lakhchaura \& Singh 2014). There are more shared populations between the BCG core and the ICL, as the ICL shares a population of old, metal rich stars with the BCG. The low mass companion has young and metal poor populations. This is consistent with an earlier stage for this cluster than for A85, where minor mergers building the BCG are contributing to the ICL. IIZw108 consists of three galaxies that are similar in mass with a high probablility of being bound, and merger timescales less than $0.1 \mathrm{Gyr}$. This is consistent with a picture where it is currently undergoing major mergers and in the process of building its BCG. There is little extended ICL, much of which shares the same high Z, low age populations as the merging galaxies, with a large contribution from young, metal poor stars that exist no where else in the system. These major mergers do not contribute to the young, metal poor component of the ICL, but part of this population will age and may be the source of the old, metal poor component in systems like A85 and A2457.

We are working on 13 additional nearby clusters (A75, A193, A376, A407, A1668, A1795, A2199, A2622, A2626, A2665, MKW3s, Zw2844, Zw8338). This is the first comprehensive sample of ICL observed spectroscopically.

\section{References}

Beers, T. C., Geller, M. J., \& Huchra, J. P. 1982, ApJ, 257, 23

Bershady, M., Anderson, M., Harker, J., Ramsey, L., \& Verheijen, M. 2004, PASP, 116, 565

Binney, J. J. \& Tremaine, S. ed 1987, Galactic Dynamics

Brough, S., Forbes, D. A., Kilborn, V. A., Couch, W., \& Colless, M. 2006, MNRAS, 369, 1351

Brough, S., Tran, K.-V., Sharp, R. G., von der Linden, A., \& Couch, W. J. 2011, MNRAS, 414, 80

Bruzual, G. \& Charlot, S. 2003, MNRAS, 344, 1000

Calzetti, D., Armus, L., Bohlin, R. C., Kinney, A. L., Koornneef, J., \& Storchi-Bergmann, T. 2000, ApJ, 533, 682

Cappellari, M., Bacon, R., Bureau, M. et al. 2006, MNRAS, 366, 1126

Chabrier, G. 2003, PASP, 115, 763

Cid Fernandes, R., Mateus, A., Sodré, L., Stasińska, G. \& Gomes, J. M. 2005, MNRAS, 358, 363

Coccatto, L, Gerhard, O. \& Arnaboldi, M. 2011, MNRAS, 407, 26

Coccatto, L, Gerhard, O., Arnaboldi, M., \& Ventimiglia, G. 2010, A\& A, 533, 138

Coelho, P., Bruzual, G., Charlot, S., Weiss, A., Barbuy, B., \& Ferguson, J. W. 2007, MNRAS, 382,498

Crawford, C. S., Allen, S. W., Ebeling, H., Edge, A. C., \& Fabian, A. C. 1999, MNRAS, 306, 857

Edwards, L. O. V., Hudson, M. J., Balogh, M. L., \& Smith, R. J. 2007, MNRAS, 379, 100

Inagaki, T., Lin, Y.-T., Huang, H.-J., Hsieh, B.-C., \& Sugiyama, N. 2015, MNRAS, 446, 1107

Lakhchaura, K. \& Singh, K. 2014, AJ, 147, 156

McDonald, M., Veilleux, S., \& Rupke, D. S. N. 2012, ApJ, 746, 153

Melnick, J., Giraud, E., Toledo, I., Selman, F., \& Quintana, H. 2012, MNRAS, 427, 850

Oliva-Altamirano, P., Brough, S., Jimmy, Kim-Vy T., Couch, W. J., McDermid, R. M., Lidman, C., von der Linden, A., \& Sharp, R. 2015, MNRAS, 449, 3347

Walcher, C. J., Coelho, P., Gallazzi, A., \& Charlot, S. 2009, MNRAS, 398, 44 